\title{
PROFILE CORRECTION FOR CROSS RUNWAYS 24 - 06 AND 30 - 12AT AIR FORCE STATION JAMNAGAR
}

\author{
MOHIT KUMAR DUBEY, IDSE
}

Assistant Garrison Engineer, Air Force Station Jamnagar

\section{ABSTRACT}

Jamnagar Air Force station has two cross runways namely 24-06 and 30-12. Both the runways are due for resurfacing hence the profile correction of both the runways as per International Civil Aviation Organization (ICAO) Annex 14 Volume III]: "Aerodrome Design and Operations" becomes an imperative task. Though the Annex 14 lays down criteria for longitudinal and transverse slope for different categories of runways, it is silent on rate of change of transverse slope along the length. Further the task of profile correction becomes more complex due to presence of intersection, since longitudinal slope of one runway becomes the transverse slope for the other. This paper thus talks about how innovative methods are used to work out the optimal quantity of Dense Bituminous Macadam (DBM) which is to be used as profile correction layer.

KEYWORDS: Dense Bituminous Macadam (DBM), Civil Aviation Organization (ICAO), Air Force

Received: Jan 05, 2021; Accepted: Jan 25, 2021; Published: Feb 26, 2021; Paper Id.: IJCSEIERDAPR20212

\section{INTRODUCTION}

Two cross runways 24-06 and 30-12 are at an angle of 60 degrees and are functional round the year. Military Engineer Services (MES) ${ }^{[3]}$ has been entrusted to undertake the resurfacing of both the runways. Runway 24-06 is $2522 \mathrm{~m}$ long and $45.72 \mathrm{~m}$ wide. It includes $426.5 \mathrm{~m}$ rigid pavement on each end and 1669 mof central flexible pavement. Runway $30-12$ is $2514 \mathrm{~m}$ long and $45.72 \mathrm{~m}$ wide. It includes $400 \mathrm{~m}$ rigid pavement on both ends and 1715 mof central flexible pavement. Code No "4" and Code letter "D" are applicable to both the runways as per “Table 1-1: Aerodrome reference code" of ICAO Annex 14 Volume I: "Aerodrome Design and Operations". As per Code No "4" and code letter "D", following are the limitations put on the longitudinal and transverse slope by $\mathrm{ICAO}^{[1]}$ :

Longitudinal slope:

- $\quad$ The slope computed by dividing the difference between the maximum and minimum elevation along the runway length should not exceed $1 \%$

- Along no portion of a runway should the longitudinal slope exceed $1.25 \%$, except that for the first and last quarter of the length of the runway the longitudinal slope should not exceed $0.8 \%$

- Where longitudinal slope changes cannot be avoided, a slope change between two consecutive slopes should not exceed $1.5 \%$

- $\quad$ The transition from one slope to another should be accomplished by a curved surface with a rate of change not exceeding $0.1 \%$ per $30 \mathrm{~m}$ (minimum radius of curvature of $30000 \mathrm{~m}$ ) 
- $\quad$ The distance between the points of intersection of two successive curves should not be less than:

- $\quad$ The sum of the absolute numerical values of the corresponding slope changes multiplied by $30000 \mathrm{~m}$

- $45 \mathrm{~m}$

Whichever is greater.

Transverse slope:

- $\quad$ To promote the most rapid drainage of water, the runway surface should, if practicable, be cambered except where a single crossfall from high to low in the direction of the wind most frequently associated with rain would ensure rapid drainage. The transverse slope should ideally be $1.5 \%$.

- But in any event should not exceed $1.5 \%$ or $2 \%$. For a cambered surface the transverse slope on each side of the centre line should be symmetrical.

- The transverse slope should be substantially the same throughout the length of the runway except at an intersection with another runway or a taxiway where an even transition should be provided taking account of the need for adequate drainage.

\section{CHALLENGES}

Challenges while working out the finished longitudinal and transverse profile of both the runways as per ICAO ${ }^{[1]}$ were as follows:

- Application of ICAO guidelines on longitudinal and transverse slopes to find out the optimal quantities especially when ICAO guidelines are silent on transition of transverse slope along the length and runway 30-12 exhibiting different types of cross sections varying from cambered (two sidesloped) to one side sloped cross section at different locations along the length.

- Meeting all the requirements of longitudinal and transverse slope becomes difficult since longitudinal slope of one runway becomes the transverse slope of the other runway at the intersection.

- $\quad$ Final quantity estimation of DBM layer.

\section{METHODOLOGY}

This section describes that how each of the above challenges were resolved. Methodology for each of the challenge is discussed as below:

- Optimization of quantity of DBM would mean the minimum quantity of DBM layer required to satisfy longitudinal as well as transverse slopes of the final proposed layer for both the runways as per ICAO ${ }^{[1][2]}$ guidelines. Topographical survey of the airfield provides the data of the existing levels in a grid of $5 \mathrm{~m} X 5 \mathrm{~m}$ i.e. at an interval of every $5 \mathrm{~m}$ along the length of the runway and at an interval of every $5 \mathrm{~m}$ on both the sides of the centreline of the runway. Profile correction is mainly a two step process, where longitudinal and transverse slopes are corrected independently. Thus the exercise on longitudinal and transverse slopes correction are discussed separately: 
- Longitudinal slope: To work out the final longitudinal profile of both the runways, sections of length $100 \mathrm{~m}$ each are taken. Parabolic curves are fitted in each of these $100 \mathrm{~m}$ sections and slope of centreline of runway are adjusted to reach the finished profile of centreline satisfying all the ICAO requirements. Final finished elevations of centreline are then used to work out the elevations along the transverse cross sections, this exercise is discussed in the next section. The exercise is done in MS Excel. Figure 1 shows the existing and proposed longitudinal profile of the runway 30-12 and Figure 2 shows the existing and proposed longitudinal profile of the runway 2406.

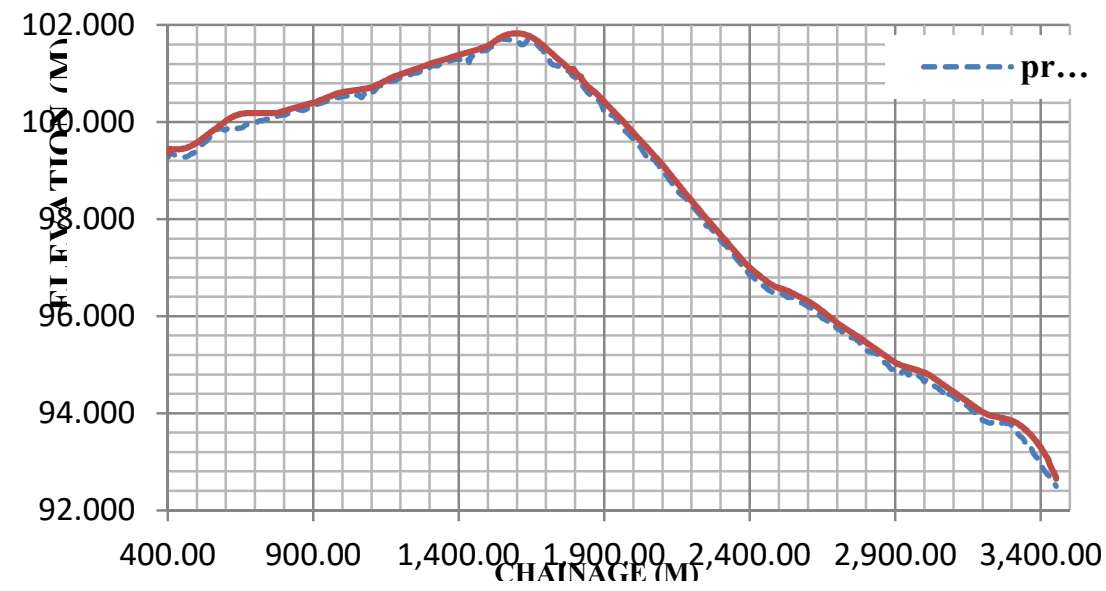

Figure 1: Existing and Proposed Longitudinal Profile of Runway 30-12

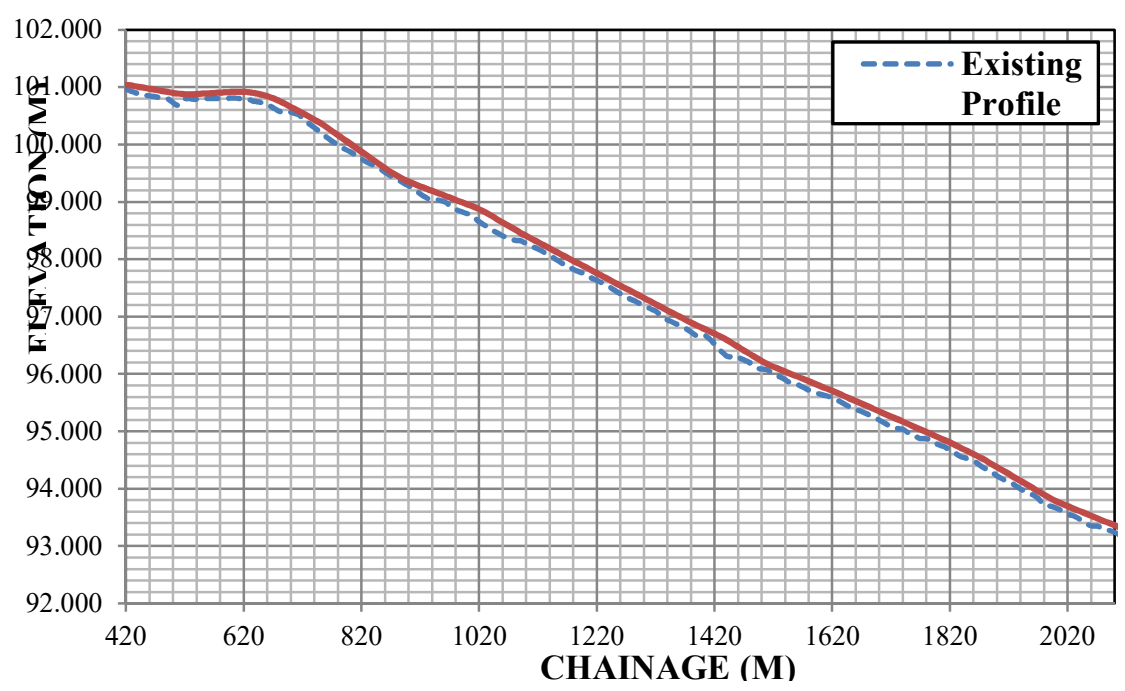

Figure 2: Existing and Proposed Longitudinal Profile of Runway 24-06

- Transverse Slope: Cross section of runway 24-06 has one side slope along the entire length as shown in Figure 3, while runway 30-12 exhibits various types of cross sections along the length. It varies from cambered cross section to one side sloped cross section as shown in Figure 4. 


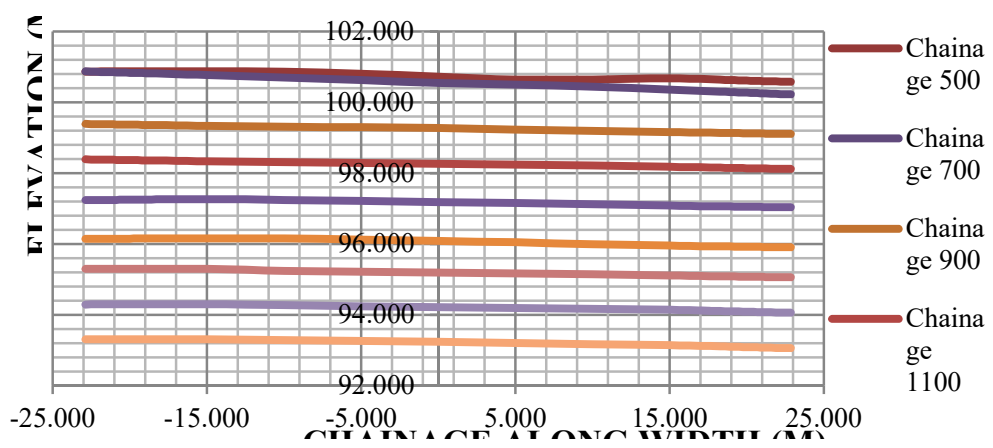

Figure 3: Existing Cross Sections of Runway 24-06 along the length

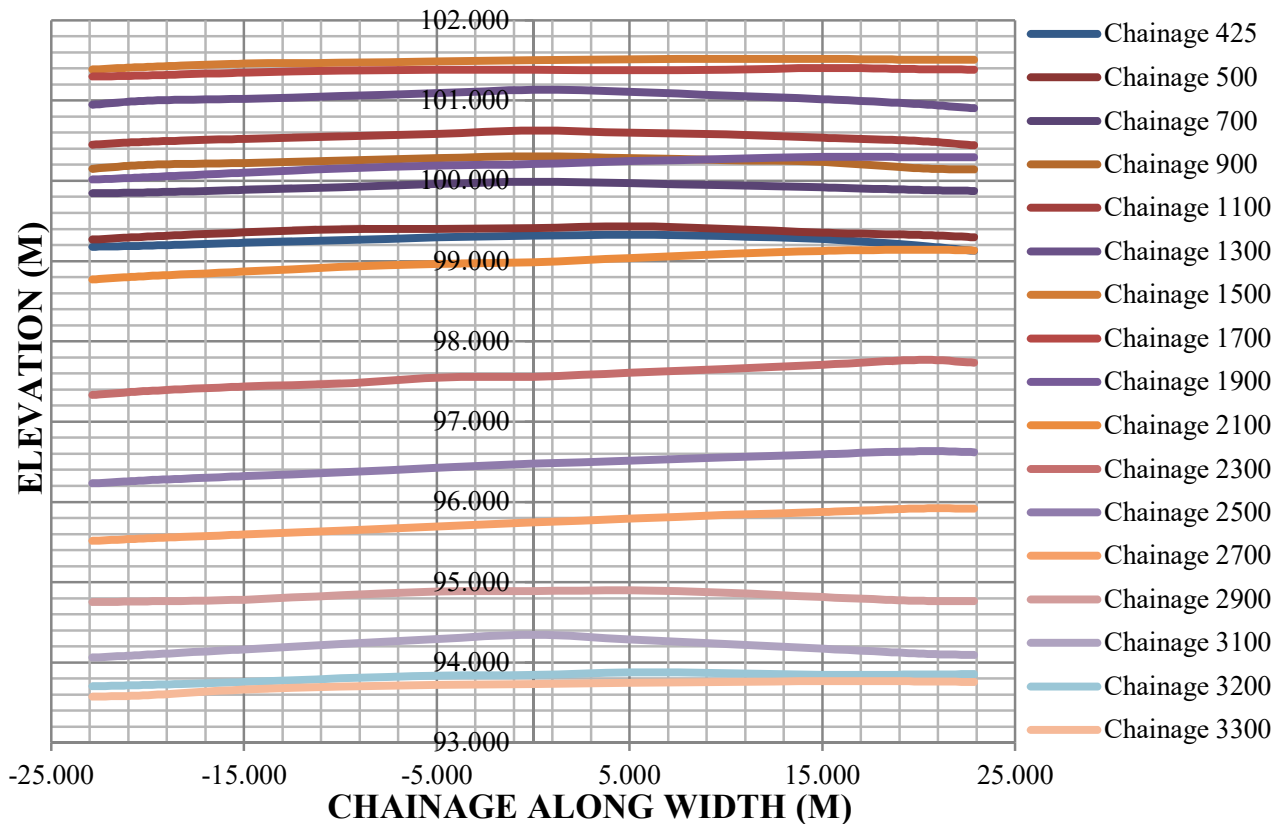

Figure 4: Existing Cross Section of Runway 30-12 along the length

To correct the transverse slope of runway 30-12 along the length of the runway, it is not financially viable that all cross sections be changed to cambered cross sections. Hence one side sloped cross sections are maintained and corrected as per ICAO guidelines ${ }^{[2]}$. Also, transition from one cross section type to other cross section is done by assuming the transition in transverse slope same as of criteria for change of slope in longitudinal slope i.e. $0.1 \%$ in $30 \mathrm{~m}$.

To correct the existing transverse slope to meet the criteria of $\mathrm{ICAO}^{[2]}$, regression analysis is performed at every cross section i.e. at every $5 \mathrm{~m}$ along the length of the runway. For this purpose width of runway is divided into two parts one on each side of centreline of runway i.e. $-22.86 \mathrm{~m}$ to 0 and from 0 to $+22.86 \mathrm{~m}$. After doing regression analysis slope of line of best fit was found on each side and then it was corrected to meet the criteria of ICAO $^{[2]}$. All the respective points across the width were found using this corrected transverse slope and the final elevation of centreline (which is in turn worked out from the correction of longitudinal slope, as described in previous section). Figure $5 \mathrm{a}$ and $5 \mathrm{~b}$ depicts this exercise for runway 30-12 at the locations of cambered cross sections. Figure $5 \mathrm{c}$ depicts this exercise at the location of one side sloped cross section. Figure 6 depicts this exercise for the runway 24-06 which has only one side sloped cross sections. 


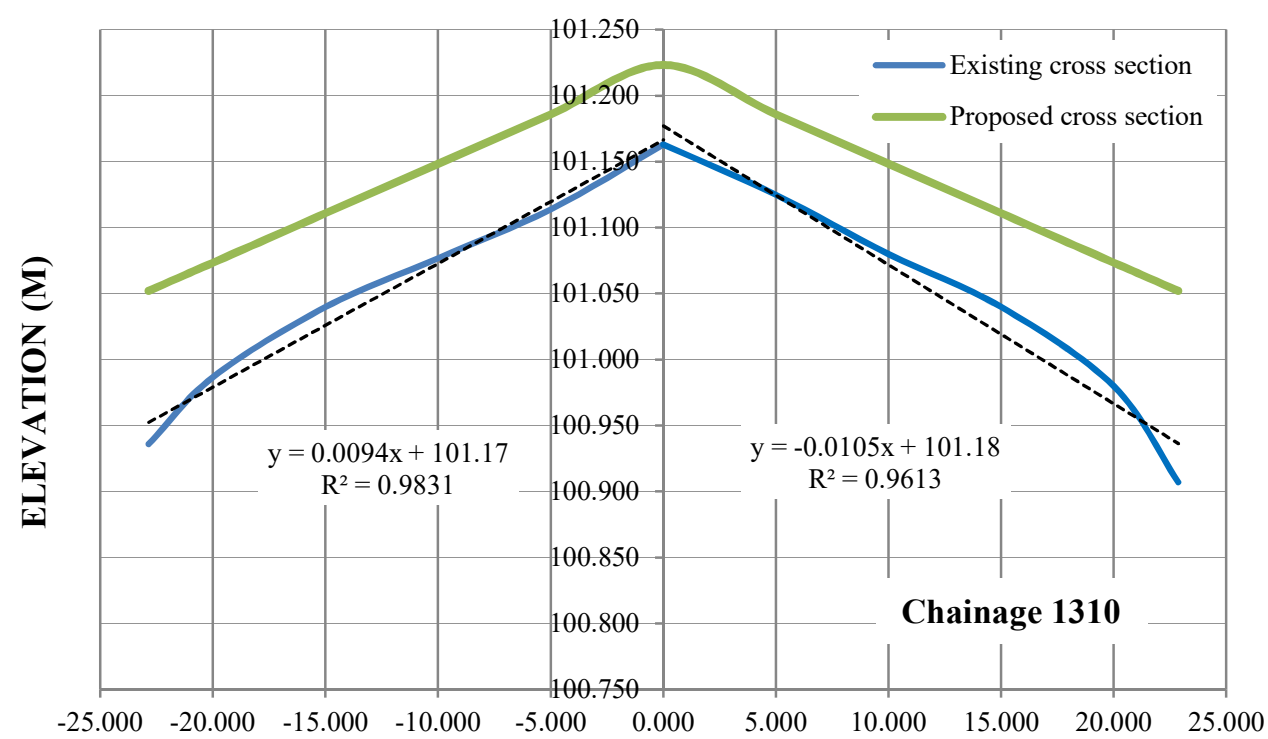

CHAINAGE ALONG WIDTH (M)

Figure 5a: Cambered Cross Section for Runway 30-12

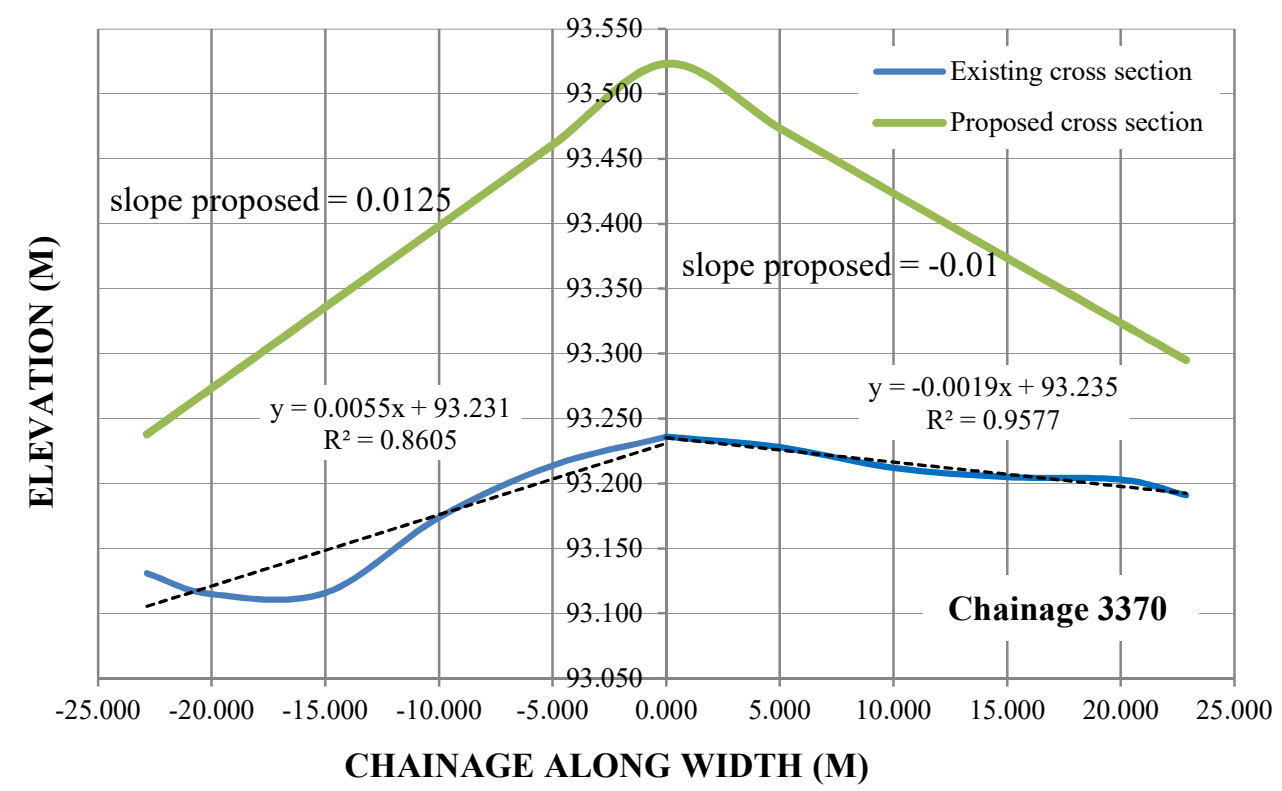

Figure 5b: Cambered Cross Section for Runway 30-12 


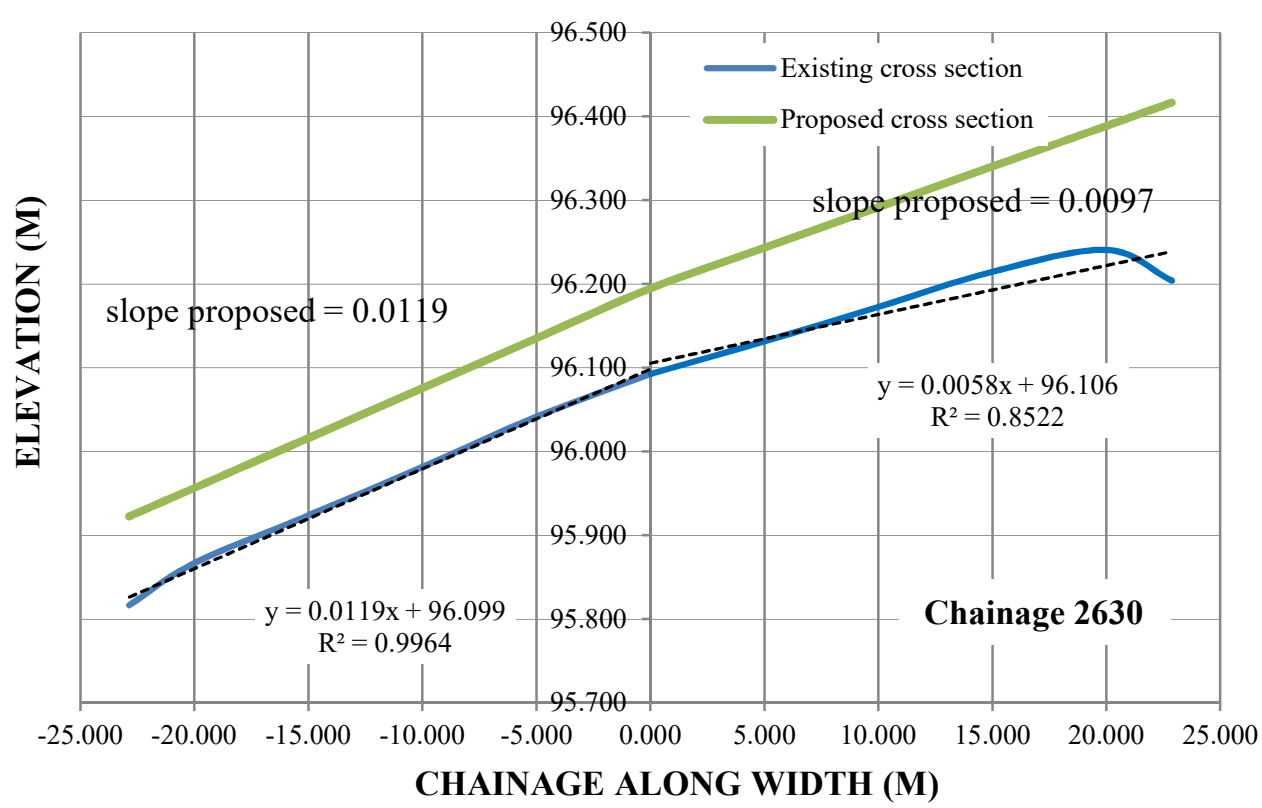

Figure 5c: One Side Sloped Cross Section for Runway 30-12

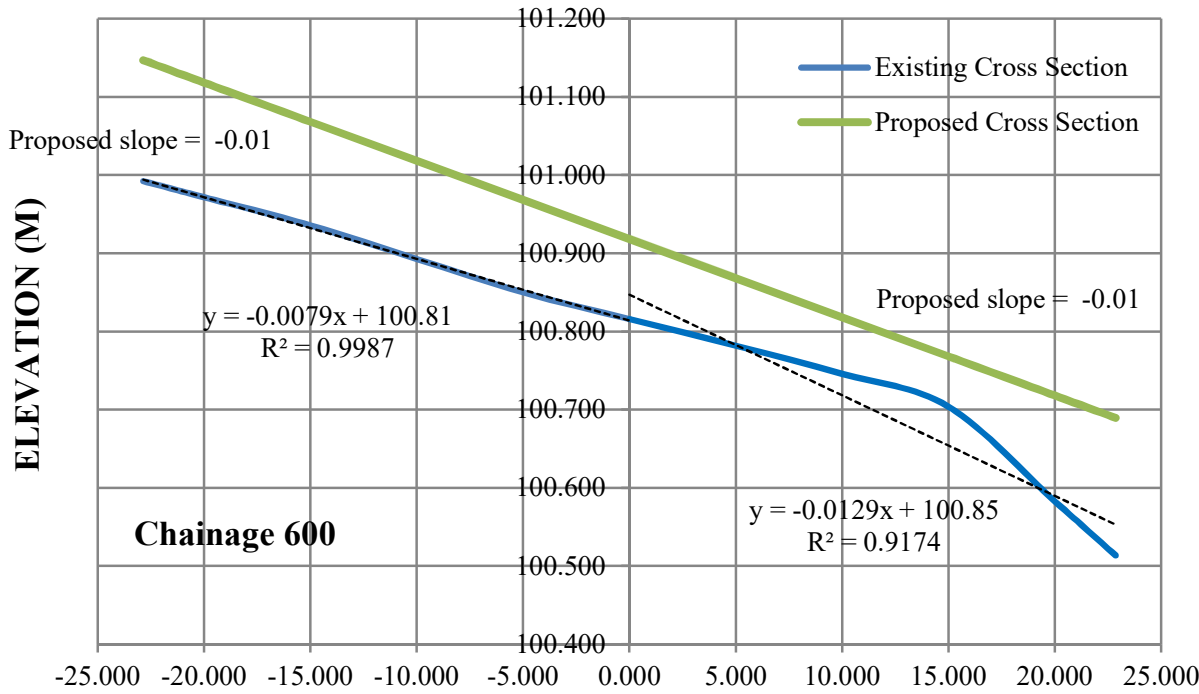

CHAINAGE ALONG WIDTH (M)

Figure 6: One Side Sloped Cross Section for Runway 24-06

- Intersection area is common for both the runways hence this area needs to be designed such that it meets the longitudinal and transverse slope guidelines of $\mathrm{ICAO}^{[1][2]}$ for both the runways. Both the runways 30-12 and 2406 have downward longitudinal slope along the length at the intersection area. Hence the transverse slope of both the runways are also one side at intersection. To design this area separately, it also becomes important to decide that which runway would govern the design of intersection area. Distances available to fighter aircraft before entering to intersection area are $600 \mathrm{~m}$ from 24 end, $1855 \mathrm{~m}$ from 06 end, $1110 \mathrm{~m}$ from 30 end and $1315 \mathrm{~m}$ from 12 end. Since the distance available is minimum from 24 end, transverse slope of 30-12 runway will be controlled by the longitudinal slope of runway 24-06 in order to avoid any major undulations in the intersection 
area when an aircraft enters from 24 end at a very high speed. In other words, runway 24-06 is treated as Primary runway while the runway 30-12 is treated as Secondary runway for the design purpose of intersection area.

Since the design of the intersection is now guided by runway 24-06, first step involves design of longitudinal and transverse slope of runway 24-06 independent of runway 30-12 (this has already been done as explained in Point no. 1). At this stage all points lying in intersection will have elevations obtained from design of runway 24-06. Second step involves transferring of final elevations (of runway 24-06, computed earlier in Point no. 1) of common points lying in the intersection region from 24-06 runway to 30-12 runway. Figure 7 shows the plan layout of intersection area and the common points to both the runways making the boundary of intersection. Third step involves using the elevations of points lying in intersection area to formulate the final elevations of points on runway 30-12 before and after the intersection area to satisfy the $\mathrm{ICAO}^{[1][2]}$ guidelines. This would again mean transition of transverse slope before and after the intersection for runway 30-12. For this purpose of transition in transverse slope is assumed same as guidelines from $\operatorname{ICAO}^{[1][2]}$ for change in longitudinal slope. Table 1 shows the elevations of runway 24-06 as worked out independent of runway 30-12. Coloured cells indicate elevations of the point lying inside the closed boundary of intersection region. Table 2 shows the elevation of points of runway 30-12 lying inside intersection region which are worked out using elevations of points of runway 24-06 lying inside intersection area. Coloured cells indicate elevations of points lying inside closed boundary of intersection region.

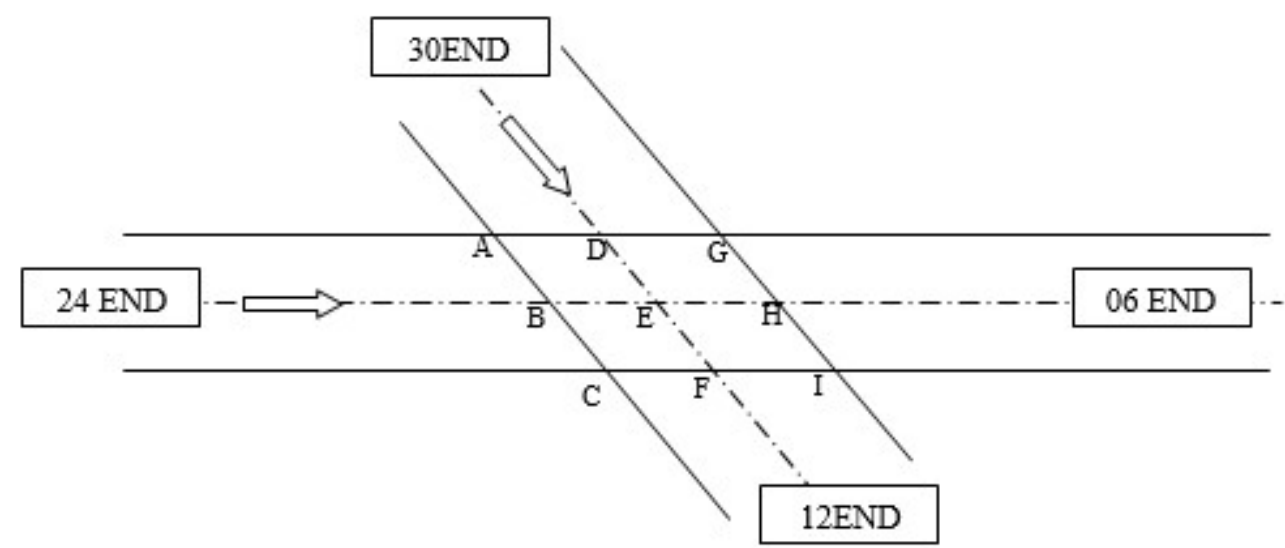

Figure 7: Plan Layout of Runway Intersection Area

Table 1: Elevations falling in the region of intersection for runway 24-06

\begin{tabular}{|c|c|c|c|c|c|c|c|c|c|c|c|c|}
\hline & $\begin{array}{c}\text { Chaina } \\
\text { ge }\end{array}$ & -22.86 & -20 & -15 & -10 & -5 & 0 & 5 & 10 & 15 & 20 & 22.86 \\
\hline $\mathbf{A}$ & 587.486 & $\begin{array}{l}100.93 \\
1\end{array}$ & $\begin{array}{l}100.97 \\
2\end{array}$ & $\begin{array}{l}101.04 \\
4\end{array}$ & $\begin{array}{l}100.90 \\
0\end{array}$ & $\begin{array}{l}100.84 \\
7\end{array}$ & $\begin{array}{l}100.80 \\
3\end{array}$ & $\begin{array}{l}100.76 \\
3\end{array}$ & $\begin{array}{l}100.72 \\
2\end{array}$ & $\begin{array}{l}100.65 \\
9\end{array}$ & $\begin{array}{l}100.57 \\
3\end{array}$ & $3^{100.52}$ \\
\hline & 590 & $\begin{array}{l}100.94 \\
5\end{array}$ & $\begin{array}{l}100.97 \\
8\end{array}$ & $\begin{array}{l}101.03 \\
6\end{array}$ & $\begin{array}{l}100.90 \\
3\end{array}$ & $\begin{array}{l}100.84 \\
9\end{array}$ & $\begin{array}{l}100.80 \\
5\end{array}$ & $\begin{array}{l}100.76 \\
6\end{array}$ & $\begin{array}{l}100.73 \\
0\end{array}$ & $\begin{array}{l}100.67 \\
6\end{array}$ & $\begin{array}{l}100.58 \\
3\end{array}$ & $\begin{array}{l}100.53 \\
0\end{array}$ \\
\hline & 595 & $\begin{array}{l}100.99 \\
9\end{array}$ & $\begin{array}{l}100.97 \\
6\end{array}$ & $\begin{array}{l}100.93 \\
5\end{array}$ & $\begin{array}{l}100.89 \\
9\end{array}$ & $\begin{array}{l}100.85 \\
3\end{array}$ & $\begin{array}{l}100.81 \\
0\end{array}$ & $\begin{array}{l}100.77 \\
4\end{array}$ & $\begin{array}{l}100.73 \\
9\end{array}$ & $\begin{array}{l}100.70 \\
1\end{array}$ & $\begin{array}{l}100.60 \\
0\end{array}$ & $\begin{array}{l}100.54 \\
2\end{array}$ \\
\hline & 600 & $\begin{array}{l}100.99 \\
3\end{array}$ & $\begin{array}{l}100.97 \\
2\end{array}$ & $\begin{array}{l}100.93 \\
6\end{array}$ & $\begin{array}{l}100.89 \\
3\end{array}$ & $\begin{array}{l}100.85 \\
0\end{array}$ & $\begin{array}{l}100.81 \\
6\end{array}$ & $\begin{array}{l}100.78 \\
2\end{array}$ & $\begin{array}{l}100.74 \\
6\end{array}$ & $\begin{array}{l}100.70 \\
4\end{array}$ & $\begin{array}{l}100.58 \\
3\end{array}$ & $\begin{array}{l}100.51 \\
4\end{array}$ \\
\hline B & 603.492 & $\begin{array}{l}100.96 \\
1\end{array}$ & $\begin{array}{l}100.95 \\
0\end{array}$ & $\begin{array}{l}100.93 \\
2\end{array}$ & $\begin{array}{l}100.89 \\
6\end{array}$ & $\begin{array}{l}100.85 \\
0\end{array}$ & $\begin{array}{l}100.81 \\
3\end{array}$ & $\begin{array}{l}100.78 \\
1\end{array}$ & $\begin{array}{l}100.75 \\
0\end{array}$ & $\begin{array}{l}100.71 \\
1\end{array}$ & $\begin{array}{l}100.59 \\
2\end{array}$ & $\begin{array}{l}100.52 \\
4\end{array}$ \\
\hline
\end{tabular}




\begin{tabular}{|c|c|c|c|c|c|c|c|c|c|c|c|c|}
\hline & 605 & $\begin{array}{l}100.94 \\
7\end{array}$ & $\begin{array}{l}100.94 \\
1\end{array}$ & $\begin{array}{l}100.93 \\
0\end{array}$ & $\begin{array}{l}100.89 \\
8\end{array}$ & $\begin{array}{l}100.85 \\
0\end{array}$ & $\begin{array}{l}100.81 \\
2\end{array}$ & $\begin{array}{l}100.78 \\
0\end{array}$ & $\begin{array}{l}100.75 \\
2\end{array}$ & $\begin{array}{l}100.71 \\
4\end{array}$ & $\begin{array}{l}100.59 \\
6\end{array}$ & $\begin{array}{l}100.52 \\
9\end{array}$ \\
\hline & 610 & $\begin{array}{l}100.96 \\
5\end{array}$ & $\begin{array}{l}100.94 \\
3\end{array}$ & $\begin{array}{l}100.90 \\
4\end{array}$ & $\begin{array}{l}100.89 \\
2\end{array}$ & $\begin{array}{l}100.85 \\
0\end{array}$ & $\begin{array}{l}100.80 \\
7\end{array}$ & $\begin{array}{l}100.77 \\
0\end{array}$ & $\begin{array}{l}100.74 \\
2\end{array}$ & $\begin{array}{l}100.70 \\
3\end{array}$ & $\begin{array}{l}100.60 \\
4\end{array}$ & $\begin{array}{l}100.54 \\
7\end{array}$ \\
\hline & 615 & $\begin{array}{l}100.95 \\
0 \\
\end{array}$ & $\begin{array}{l}100.93 \\
9\end{array}$ & $\begin{array}{l}100.91 \\
9\end{array}$ & $\begin{array}{l}100.88 \\
3\end{array}$ & $\begin{array}{l}100.84 \\
8 \\
\end{array}$ & $\begin{array}{l}100.80 \\
1\end{array}$ & $\begin{array}{l}100.76 \\
8\end{array}$ & $\begin{array}{l}100.74 \\
7 \\
\end{array}$ & $\begin{array}{l}100.70 \\
1\end{array}$ & $\begin{array}{l}100.65 \\
0 \\
\end{array}$ & $\begin{array}{l}100.62 \\
1 \\
\end{array}$ \\
\hline C & 619.5 & $\begin{array}{l}100.95 \\
4\end{array}$ & $\begin{array}{l}100.94 \\
4\end{array}$ & $\begin{array}{l}100.92 \\
5\end{array}$ & $\begin{array}{l}100.88 \\
5\end{array}$ & $\begin{array}{l}100.84 \\
0\end{array}$ & $\begin{array}{l}100.79 \\
7\end{array}$ & $\begin{array}{l}100.76 \\
1\end{array}$ & $\begin{array}{l}100.74 \\
3\end{array}$ & $\begin{array}{l}100.69 \\
8\end{array}$ & $\begin{array}{l}100.64 \\
0\end{array}$ & $\begin{array}{l}100.60 \\
7\end{array}$ \\
\hline & 620 & $\begin{array}{l}100.95 \\
4\end{array}$ & $\begin{array}{l}100.94 \\
4\end{array}$ & $\begin{array}{l}100.92 \\
6\end{array}$ & $\begin{array}{l}100.88 \\
5\end{array}$ & $\begin{array}{l}100.83 \\
9\end{array}$ & $\begin{array}{l}100.79 \\
6\end{array}$ & $\begin{array}{l}100.76 \\
0\end{array}$ & $\begin{array}{l}100.74 \\
3\end{array}$ & $\begin{array}{l}100.69 \\
8\end{array}$ & $\begin{array}{l}100.63 \\
9\end{array}$ & $\begin{array}{l}100.60 \\
5\end{array}$ \\
\hline & 625 & $\begin{array}{l}100.95 \\
7\end{array}$ & $\begin{array}{l}100.94 \\
4\end{array}$ & $\begin{array}{l}100.92 \\
2\end{array}$ & $\begin{array}{l}100.87 \\
4\end{array}$ & $\begin{array}{l}100.83 \\
0\end{array}$ & $\begin{array}{l}100.79 \\
0\end{array}$ & $\begin{array}{l}100.75 \\
4\end{array}$ & $\begin{array}{l}100.72 \\
3\end{array}$ & $\begin{array}{l}100.69 \\
1\end{array}$ & $\begin{array}{l}100.67 \\
6\end{array}$ & $\begin{array}{l}100.66 \\
7\end{array}$ \\
\hline & 630 & $\begin{array}{l}100.95 \\
4\end{array}$ & $\begin{array}{l}100.94 \\
3\end{array}$ & $\begin{array}{l}100.92 \\
4\end{array}$ & $\begin{array}{l}100.87 \\
4\end{array}$ & $\begin{array}{l}100.81 \\
7\end{array}$ & $\begin{array}{l}100.78 \\
3\end{array}$ & $\begin{array}{l}100.74 \\
7\end{array}$ & $\begin{array}{l}100.71 \\
1\end{array}$ & $\begin{array}{l}100.67 \\
7\end{array}$ & $\begin{array}{l}100.65 \\
7\end{array}$ & $\begin{array}{l}100.64 \\
6\end{array}$ \\
\hline $\mathbf{E}$ & 631.399 & $\begin{array}{l}100.95 \\
2\end{array}$ & $\begin{array}{l}100.94 \\
2\end{array}$ & $\begin{array}{l}100.92 \\
4\end{array}$ & $\begin{array}{l}100.87 \\
4\end{array}$ & $\begin{array}{l}100.81 \\
6\end{array}$ & $\begin{array}{l}100.77 \\
7\end{array}$ & $\begin{array}{l}100.74 \\
3\end{array}$ & $\begin{array}{l}100.70 \\
8\end{array}$ & $\begin{array}{l}100.67 \\
2\end{array}$ & $\begin{array}{l}100.64 \\
9\end{array}$ & $\begin{array}{l}100.63 \\
7\end{array}$ \\
\hline & 635 & $\begin{array}{l}100.94 \\
6\end{array}$ & $\begin{array}{l}100.93 \\
8\end{array}$ & $\begin{array}{l}100.92 \\
4\end{array}$ & $\begin{array}{l}100.87 \\
5\end{array}$ & $\begin{array}{l}100.81 \\
4\end{array}$ & $\begin{array}{l}100.76 \\
2\end{array}$ & $\begin{array}{l}100.73 \\
3\end{array}$ & $\begin{array}{l}100.70 \\
0\end{array}$ & $\begin{array}{l}100.65 \\
8\end{array}$ & $\begin{array}{l}100.63 \\
0\end{array}$ & $\begin{array}{l}100.61 \\
4\end{array}$ \\
\hline & 640 & $\begin{array}{l}100.94 \\
1 \\
\end{array}$ & $\begin{array}{l}100.93 \\
2\end{array}$ & $\begin{array}{l}100.91 \\
6\end{array}$ & $\begin{array}{l}100.87 \\
3\end{array}$ & $\begin{array}{l}100.81 \\
3\end{array}$ & $\begin{array}{l}100.75 \\
2\end{array}$ & $\begin{array}{l}100.70 \\
9\end{array}$ & $\begin{array}{l}100.68 \\
0\end{array}$ & $\begin{array}{l}100.64 \\
4 \\
\end{array}$ & $\begin{array}{l}100.60 \\
4\end{array}$ & $\begin{array}{l}100.58 \\
1\end{array}$ \\
\hline G & 643.299 & $\begin{array}{l}100.92 \\
6 \\
\end{array}$ & $\begin{array}{l}100.91 \\
4 \\
\end{array}$ & $\begin{array}{l}100.89 \\
4\end{array}$ & $\begin{array}{l}100.86 \\
0\end{array}$ & $\begin{array}{l}100.80 \\
3 \\
\end{array}$ & $\begin{array}{l}100.74 \\
7\end{array}$ & $\begin{array}{l}100.69 \\
6 \\
\end{array}$ & $\begin{array}{l}100.66 \\
4 \\
\end{array}$ & $\begin{array}{l}100.63 \\
5 \\
\end{array}$ & $\begin{array}{l}100.60 \\
4\end{array}$ & $\begin{array}{l}100.58 \\
6 \\
\end{array}$ \\
\hline & 645 & $\begin{array}{l}100.91 \\
8\end{array}$ & $\begin{array}{l}100.90 \\
5\end{array}$ & $\begin{array}{l}100.88 \\
3\end{array}$ & $\begin{array}{l}100.85 \\
4\end{array}$ & $\begin{array}{l}100.79 \\
8\end{array}$ & $\begin{array}{l}100.74 \\
5\end{array}$ & $\begin{array}{l}100.68 \\
9\end{array}$ & $\begin{array}{l}100.65 \\
5\end{array}$ & $\begin{array}{l}100.63 \\
0\end{array}$ & $\begin{array}{l}100.60 \\
4\end{array}$ & $\begin{array}{l}100.58 \\
9\end{array}$ \\
\hline & 650 & $\begin{array}{l}100.89 \\
8\end{array}$ & $\begin{array}{l}100.88 \\
4\end{array}$ & $\begin{array}{l}100.86 \\
0\end{array}$ & $\begin{array}{l}100.82 \\
4\end{array}$ & $\begin{array}{l}100.78 \\
1\end{array}$ & $\begin{array}{l}100.73 \\
1\end{array}$ & $\begin{array}{l}100.68 \\
0\end{array}$ & $\begin{array}{l}100.63 \\
2\end{array}$ & $\begin{array}{l}100.60 \\
5\end{array}$ & $\begin{array}{l}100.59 \\
8\end{array}$ & $\begin{array}{l}100.59 \\
4\end{array}$ \\
\hline & 655 & $\begin{array}{l}100.88 \\
1 \\
\end{array}$ & $\begin{array}{l}100.86 \\
3\end{array}$ & $\begin{array}{l}100.83 \\
1\end{array}$ & $\begin{array}{l}100.79 \\
0\end{array}$ & $\begin{array}{l}100.74 \\
7\end{array}$ & $\begin{array}{l}100.70 \\
7\end{array}$ & $\begin{array}{l}100.66 \\
1\end{array}$ & $\begin{array}{l}100.61 \\
3\end{array}$ & $\begin{array}{l}100.57 \\
8\end{array}$ & $\begin{array}{l}100.57 \\
1\end{array}$ & $\begin{array}{l}100.56 \\
7\end{array}$ \\
\hline H & 659.306 & $\begin{array}{l}100.86 \\
3\end{array}$ & $\begin{array}{l}100.84 \\
1\end{array}$ & $\begin{array}{l}100.80 \\
3\end{array}$ & $\begin{array}{l}100.77 \\
0\end{array}$ & $\begin{array}{l}100.74 \\
4\end{array}$ & $\begin{array}{l}100.69 \\
8\end{array}$ & $\begin{array}{l}100.62 \\
9\end{array}$ & $\begin{array}{l}100.59 \\
6\end{array}$ & $\begin{array}{l}100.55 \\
6\end{array}$ & $\begin{array}{l}100.54 \\
4\end{array}$ & $\begin{array}{l}100.53 \\
8\end{array}$ \\
\hline & 660 & $\begin{array}{l}100.86 \\
0\end{array}$ & $\begin{array}{l}100.83 \\
8\end{array}$ & $\begin{array}{l}100.79 \\
9\end{array}$ & $\begin{array}{l}100.76 \\
7\end{array}$ & $\begin{array}{l}100.74 \\
4\end{array}$ & $\begin{array}{l}100.69 \\
7\end{array}$ & $\begin{array}{l}100.62 \\
4\end{array}$ & $\begin{array}{l}100.59 \\
3\end{array}$ & $\begin{array}{l}100.55 \\
2\end{array}$ & $\begin{array}{l}100.54 \\
0\end{array}$ & $\begin{array}{l}100.53 \\
3\end{array}$ \\
\hline & 665 & $\begin{array}{l}100.82 \\
8\end{array}$ & $\begin{array}{l}100.81 \\
5\end{array}$ & $\begin{array}{l}100.79 \\
2\end{array}$ & $\begin{array}{l}100.77 \\
0\end{array}$ & $\begin{array}{l}100.74 \\
2\end{array}$ & $\begin{array}{l}100.67 \\
0\end{array}$ & $\begin{array}{l}100.58 \\
6\end{array}$ & $\begin{array}{l}100.55 \\
2\end{array}$ & $\begin{array}{l}100.52 \\
3\end{array}$ & $\begin{array}{l}100.49 \\
3\end{array}$ & $\begin{array}{l}100.47 \\
6\end{array}$ \\
\hline & 670 & $\begin{array}{l}100.83 \\
0\end{array}$ & $\begin{array}{l}100.81 \\
7\end{array}$ & $\begin{array}{l}100.79 \\
5\end{array}$ & $\begin{array}{l}100.77 \\
4\end{array}$ & $\begin{array}{l}100.72 \\
8\end{array}$ & $\begin{array}{l}100.64 \\
1\end{array}$ & $\begin{array}{l}100.55 \\
1\end{array}$ & $\begin{array}{l}100.51 \\
0\end{array}$ & $\begin{array}{l}100.51 \\
0\end{array}$ & $\begin{array}{l}100.44 \\
5\end{array}$ & $\begin{array}{l}100.40 \\
8\end{array}$ \\
\hline & 675 & $\begin{array}{l}100.84 \\
2\end{array}$ & $\begin{array}{l}100.82 \\
7\end{array}$ & $\begin{array}{l}100.80 \\
0\end{array}$ & $\begin{array}{l}100.77 \\
9\end{array}$ & $\begin{array}{l}100.71 \\
2\end{array}$ & $\begin{array}{l}100.61 \\
2\end{array}$ & $\begin{array}{l}100.51 \\
7\end{array}$ & $\begin{array}{l}100.50 \\
7\end{array}$ & $\begin{array}{l}100.49 \\
6\end{array}$ & $\begin{array}{l}100.42 \\
7\end{array}$ & $\begin{array}{l}100.38 \\
8\end{array}$ \\
\hline I & 675.313 & $\begin{array}{l}100.84 \\
7 \\
\end{array}$ & $\begin{array}{l}100.83 \\
0\end{array}$ & $\begin{array}{l}100.80 \\
0\end{array}$ & $\begin{array}{l}100.77 \\
8\end{array}$ & $\begin{array}{l}100.71 \\
0\end{array}$ & $\begin{array}{l}100.61 \\
0\end{array}$ & $\begin{array}{l}100.51 \\
5\end{array}$ & $\begin{array}{l}100.50 \\
7 \\
\end{array}$ & $\begin{array}{l}100.49 \\
5\end{array}$ & $\begin{array}{l}100.42 \\
3\end{array}$ & $\begin{array}{l}100.38 \\
2\end{array}$ \\
\hline
\end{tabular}

Table 2: Elevations falling in the region of intersection for runway 30-12

\begin{tabular}{|c|c|c|c|c|c|c|c|c|c|c|c|c|}
\hline & $\begin{array}{c}\text { Chaina } \\
\text { ge }\end{array}$ & -22.86 & -20 & -15 & -10 & -5 & 0 & 5 & 10 & 15 & 20 & 22.86 \\
\hline A & 1775.47 & $\begin{array}{l}101.05 \\
2\end{array}$ & $\begin{array}{l}101.06 \\
0\end{array}$ & $\begin{array}{l}101.07 \\
5\end{array}$ & $\begin{array}{l}101.09 \\
0\end{array}$ & $\begin{array}{l}101.10 \\
5\end{array}$ & $\begin{array}{l}101.12 \\
0\end{array}$ & $\begin{array}{l}101.06 \\
2\end{array}$ & $\begin{array}{l}101.00 \\
4\end{array}$ & $\begin{array}{l}100.94 \\
6\end{array}$ & $\begin{array}{l}100.88 \\
8\end{array}$ & $\begin{array}{l}101.19 \\
2\end{array}$ \\
\hline & 1780 & $\begin{array}{l}101.11 \\
7\end{array}$ & $\begin{array}{l}101.11 \\
5\end{array}$ & $\begin{array}{l}101.11 \\
1\end{array}$ & $\begin{array}{l}101.10 \\
7\end{array}$ & $\begin{array}{l}101.10 \\
3\end{array}$ & $\begin{array}{l}101.09 \\
9\end{array}$ & $\begin{array}{l}101.08 \\
6\end{array}$ & $\begin{array}{l}101.07 \\
3\end{array}$ & $\begin{array}{l}101.06 \\
0\end{array}$ & $\begin{array}{l}101.14 \\
2\end{array}$ & $\begin{array}{l}101.14 \\
0\end{array}$ \\
\hline & 1785 & $\begin{array}{l}101.10 \\
8\end{array}$ & $\begin{array}{l}101.10 \\
4\end{array}$ & $\begin{array}{l}101.09 \\
6\end{array}$ & $\begin{array}{l}101.08 \\
8\end{array}$ & $\begin{array}{l}101.08 \\
0\end{array}$ & $\begin{array}{l}101.07 \\
2\end{array}$ & $\begin{array}{l}101.06 \\
7\end{array}$ & $\begin{array}{l}101.14 \\
5\end{array}$ & $\begin{array}{l}101.11 \\
4\end{array}$ & $\begin{array}{l}101.08 \\
3\end{array}$ & $\begin{array}{l}101.07 \\
7\end{array}$ \\
\hline & 1790 & $\begin{array}{l}101.08 \\
1 \\
\end{array}$ & $\begin{array}{l}101.07 \\
6 \\
\end{array}$ & $\begin{array}{l}101.06 \\
8\end{array}$ & $\begin{array}{l}101.06 \\
0\end{array}$ & $\begin{array}{l}101.05 \\
2\end{array}$ & $\begin{array}{l}101.04 \\
5 \\
\end{array}$ & $\begin{array}{l}101.13 \\
1 \\
\end{array}$ & $\begin{array}{l}101.10 \\
5\end{array}$ & $\begin{array}{l}101.07 \\
5 \\
\end{array}$ & $\begin{array}{l}101.04 \\
3 \\
\end{array}$ & $\begin{array}{l}101.02 \\
6 \\
\end{array}$ \\
\hline D & $\begin{array}{l}1791.47 \\
6\end{array}$ & $\begin{array}{l}101.14 \\
2\end{array}$ & $\begin{array}{l}101.13 \\
8\end{array}$ & $\begin{array}{l}101.12 \\
9\end{array}$ & $\begin{array}{l}101.12 \\
1\end{array}$ & $\begin{array}{l}101.11 \\
2\end{array}$ & $\begin{array}{l}101.10 \\
4\end{array}$ & $\begin{array}{l}101.11 \\
2\end{array}$ & $\begin{array}{l}101.09 \\
3\end{array}$ & $\begin{array}{l}101.06 \\
3\end{array}$ & $\begin{array}{l}101.03 \\
2\end{array}$ & $\begin{array}{l}101.01 \\
4\end{array}$ \\
\hline & 1795 & $\begin{array}{l}101.09 \\
6\end{array}$ & $\begin{array}{l}101.09 \\
1\end{array}$ & $\begin{array}{l}101.08 \\
1\end{array}$ & $\begin{array}{l}101.07 \\
2\end{array}$ & $\begin{array}{l}101.06 \\
2\end{array}$ & $\begin{array}{l}101.08 \\
3\end{array}$ & $\begin{array}{l}101.07 \\
0\end{array}$ & $\begin{array}{l}101.06 \\
5\end{array}$ & $\begin{array}{l}101.03 \\
5\end{array}$ & $\begin{array}{l}101.00 \\
4\end{array}$ & $\begin{array}{l}100.98 \\
6 \\
\end{array}$ \\
\hline
\end{tabular}




\begin{tabular}{|c|c|c|c|c|c|c|c|c|c|c|c|c|}
\hline & 1800 & $\begin{array}{l}101.08 \\
5\end{array}$ & $\begin{array}{l}101.08 \\
1\end{array}$ & $\begin{array}{l}101.07 \\
3\end{array}$ & $\begin{array}{l}101.06 \\
6\end{array}$ & $\begin{array}{l}101.03 \\
7\end{array}$ & $\begin{array}{l}101.05 \\
1\end{array}$ & $\begin{array}{l}101.02 \\
6\end{array}$ & $\begin{array}{l}101.01 \\
6\end{array}$ & $\begin{array}{l}100.99 \\
5\end{array}$ & $\begin{array}{l}100.96 \\
5\end{array}$ & $\begin{array}{l}100.94 \\
7\end{array}$ \\
\hline & 1805 & $\begin{array}{l}101.07 \\
1\end{array}$ & $\begin{array}{l}101.06 \\
5\end{array}$ & $\begin{array}{l}101.05 \\
5\end{array}$ & $\begin{array}{l}101.04 \\
0\end{array}$ & $\begin{array}{l}101.01 \\
2\end{array}$ & $\begin{array}{l}101.01 \\
0\end{array}$ & $\begin{array}{l}100.99 \\
4\end{array}$ & $\begin{array}{l}100.97 \\
1\end{array}$ & $\begin{array}{l}100.95 \\
4\end{array}$ & $\begin{array}{l}100.92 \\
5\end{array}$ & $\begin{array}{l}100.90 \\
8\end{array}$ \\
\hline \multirow[t]{3}{*}{ G } & $\begin{array}{l}1807.48 \\
3\end{array}$ & $\begin{array}{l}101.06 \\
4\end{array}$ & $\begin{array}{l}101.05 \\
1\end{array}$ & $\begin{array}{l}101.03 \\
7\end{array}$ & $\begin{array}{l}101.02 \\
3\end{array}$ & $\begin{array}{l}100.99 \\
9\end{array}$ & $\begin{array}{l}100.99 \\
1\end{array}$ & $\begin{array}{l}100.97 \\
7\end{array}$ & $\begin{array}{l}100.95 \\
5\end{array}$ & $\begin{array}{l}100.93 \\
3\end{array}$ & $\begin{array}{l}100.90 \\
4\end{array}$ & $\begin{array}{l}100.88 \\
8\end{array}$ \\
\hline & 1810 & $\begin{array}{l}101.03 \\
9\end{array}$ & $\begin{array}{l}101.03 \\
2\end{array}$ & $\begin{array}{l}101.01 \\
9\end{array}$ & $\begin{array}{l}101.00 \\
5\end{array}$ & $\begin{array}{l}100.98 \\
6\end{array}$ & $\begin{array}{l}100.97 \\
4\end{array}$ & $\begin{array}{l}100.95 \\
8\end{array}$ & $\begin{array}{l}100.93 \\
8\end{array}$ & $\begin{array}{l}100.91 \\
5\end{array}$ & $\begin{array}{l}100.88 \\
1\end{array}$ & $\begin{array}{l}100.86 \\
5 \\
\end{array}$ \\
\hline & 1815 & $\begin{array}{l}100.99 \\
9\end{array}$ & $\begin{array}{l}100.99 \\
4\end{array}$ & $\begin{array}{l}100.98 \\
3\end{array}$ & $\begin{array}{l}100.96 \\
9\end{array}$ & $\begin{array}{l}100.95 \\
5\end{array}$ & $\begin{array}{l}100.94 \\
0\end{array}$ & $\begin{array}{l}100.92 \\
2\end{array}$ & $\begin{array}{l}100.90 \\
3\end{array}$ & $\begin{array}{l}100.88 \\
2\end{array}$ & $\begin{array}{l}100.84 \\
9\end{array}$ & $\begin{array}{l}100.81 \\
9\end{array}$ \\
\hline \multirow[t]{4}{*}{$\mathbf{E}$} & $\begin{array}{l}1819.38 \\
3\end{array}$ & $\begin{array}{l}100.96 \\
6\end{array}$ & $\begin{array}{l}100.96 \\
0\end{array}$ & $\begin{array}{l}100.95 \\
2\end{array}$ & $\begin{array}{l}100.93 \\
8\end{array}$ & $\begin{array}{l}100.92 \\
3\end{array}$ & $\begin{array}{l}100.94 \\
6\end{array}$ & $\begin{array}{l}100.89 \\
2\end{array}$ & $\begin{array}{l}100.87 \\
4\end{array}$ & $\begin{array}{l}100.85 \\
1\end{array}$ & $\begin{array}{l}100.83 \\
3\end{array}$ & $\begin{array}{l}100.80 \\
1\end{array}$ \\
\hline & 1820 & $\begin{array}{l}100.96 \\
1\end{array}$ & $\begin{array}{l}100.95 \\
6\end{array}$ & $\begin{array}{l}100.94 \\
7\end{array}$ & $\begin{array}{l}100.93 \\
3\end{array}$ & $\begin{array}{l}100.91 \\
9\end{array}$ & $\begin{array}{l}100.90 \\
4\end{array}$ & $\begin{array}{l}100.88 \\
8\end{array}$ & $\begin{array}{l}100.87 \\
0\end{array}$ & $\begin{array}{l}100.84 \\
7\end{array}$ & $\begin{array}{l}100.82 \\
8\end{array}$ & $\begin{array}{l}100.79 \\
9\end{array}$ \\
\hline & 1825 & $\begin{array}{l}100.92 \\
6\end{array}$ & $\begin{array}{l}100.91 \\
9\end{array}$ & $\begin{array}{l}100.90 \\
8\end{array}$ & $\begin{array}{l}100.89 \\
6\end{array}$ & $\begin{array}{l}100.88 \\
3\end{array}$ & $\begin{array}{l}100.86 \\
5\end{array}$ & $\begin{array}{l}100.85 \\
1\end{array}$ & $\begin{array}{l}100.83 \\
6\end{array}$ & $\begin{array}{l}100.81 \\
5\end{array}$ & $\begin{array}{l}100.79 \\
2\end{array}$ & $\begin{array}{l}100.78 \\
2\end{array}$ \\
\hline & 1830 & $\begin{array}{l}100.88 \\
7\end{array}$ & $\begin{array}{l}100.88 \\
2\end{array}$ & $\begin{array}{l}100.87 \\
1\end{array}$ & $\begin{array}{l}100.86 \\
0\end{array}$ & $\begin{array}{l}100.84 \\
8\end{array}$ & $\begin{array}{l}100.83 \\
0\end{array}$ & $\begin{array}{l}100.82 \\
1\end{array}$ & $\begin{array}{l}100.79 \\
8\end{array}$ & $\begin{array}{l}100.78 \\
7\end{array}$ & $\begin{array}{l}100.75 \\
8\end{array}$ & $\begin{array}{l}100.74 \\
4\end{array}$ \\
\hline \multirow[t]{4}{*}{ C } & $\begin{array}{l}1831.28 \\
4\end{array}$ & $\begin{array}{l}100.87 \\
6\end{array}$ & $\begin{array}{l}100.87 \\
2\end{array}$ & $\begin{array}{l}100.86 \\
1\end{array}$ & $\begin{array}{l}100.85 \\
0\end{array}$ & $\begin{array}{l}100.83 \\
8\end{array}$ & $\begin{array}{l}100.82 \\
2\end{array}$ & $\begin{array}{l}100.81 \\
4\end{array}$ & $\begin{array}{l}100.78 \\
8\end{array}$ & $\begin{array}{l}100.77 \\
7\end{array}$ & $\begin{array}{l}100.75 \\
1\end{array}$ & $\begin{array}{l}100.71 \\
7\end{array}$ \\
\hline & 1835 & $\begin{array}{l}100.84 \\
7\end{array}$ & $\begin{array}{l}100.84 \\
2\end{array}$ & $\begin{array}{l}100.83 \\
3\end{array}$ & $\begin{array}{l}100.82 \\
3\end{array}$ & $\begin{array}{l}100.81 \\
0\end{array}$ & $\begin{array}{l}100.79 \\
9\end{array}$ & $\begin{array}{l}100.79 \\
6\end{array}$ & $\begin{array}{l}100.76 \\
3\end{array}$ & $\begin{array}{l}100.74 \\
7\end{array}$ & $\begin{array}{l}100.73 \\
2\end{array}$ & $\begin{array}{l}100.72 \\
4\end{array}$ \\
\hline & 1840 & $\begin{array}{l}100.80 \\
8\end{array}$ & $\begin{array}{l}100.80 \\
6 \\
\end{array}$ & $\begin{array}{l}100.79 \\
3 \\
\end{array}$ & $\begin{array}{l}100.78 \\
5\end{array}$ & $\begin{array}{l}100.77 \\
5 \\
\end{array}$ & $\begin{array}{l}100.76 \\
2\end{array}$ & $\begin{array}{l}100.76 \\
6 \\
\end{array}$ & $\begin{array}{l}100.73 \\
9 \\
\end{array}$ & $\begin{array}{l}100.72 \\
4\end{array}$ & $\begin{array}{l}100.70 \\
9\end{array}$ & $\begin{array}{l}100.70 \\
0 \\
\end{array}$ \\
\hline & 1845 & $\begin{array}{l}100.76 \\
4\end{array}$ & $\begin{array}{l}100.76 \\
4\end{array}$ & $\begin{array}{l}100.75 \\
9\end{array}$ & $\begin{array}{l}100.74 \\
3\end{array}$ & $\begin{array}{l}100.74 \\
0\end{array}$ & $\begin{array}{l}100.72 \\
6\end{array}$ & $\begin{array}{l}100.71 \\
1\end{array}$ & $\begin{array}{l}100.69 \\
6\end{array}$ & $\begin{array}{l}100.68 \\
1\end{array}$ & $\begin{array}{l}100.66 \\
6\end{array}$ & $\begin{array}{l}100.65 \\
7\end{array}$ \\
\hline \multirow[t]{4}{*}{$\mathbf{F}$} & 1847.29 & $\begin{array}{l}100.74 \\
1\end{array}$ & $\begin{array}{l}100.74 \\
2\end{array}$ & $\begin{array}{l}100.74 \\
5\end{array}$ & $\begin{array}{l}100.72 \\
6\end{array}$ & $\begin{array}{l}100.72 \\
0\end{array}$ & $\begin{array}{l}100.69 \\
5\end{array}$ & $\begin{array}{l}100.67 \\
4\end{array}$ & $\begin{array}{l}100.65 \\
4\end{array}$ & $\begin{array}{l}100.63 \\
3\end{array}$ & $\begin{array}{l}100.61 \\
3\end{array}$ & $\begin{array}{l}100.60 \\
1\end{array}$ \\
\hline & 1850 & $\begin{array}{l}100.71 \\
3\end{array}$ & $\begin{array}{l}100.71 \\
5 \\
\end{array}$ & $\begin{array}{l}100.72 \\
1\end{array}$ & $\begin{array}{l}100.70 \\
9\end{array}$ & $\begin{array}{l}100.68 \\
4\end{array}$ & $\begin{array}{l}100.71 \\
0 \\
\end{array}$ & $\begin{array}{l}100.69 \\
5 \\
\end{array}$ & $\begin{array}{l}100.68 \\
0\end{array}$ & $\begin{array}{l}100.66 \\
5 \\
\end{array}$ & $\begin{array}{l}100.65 \\
0 \\
\end{array}$ & $\begin{array}{l}100.64 \\
1 \\
\end{array}$ \\
\hline & 1855 & $\begin{array}{l}100.65 \\
7 \\
\end{array}$ & $\begin{array}{l}100.66 \\
0\end{array}$ & $\begin{array}{l}100.66 \\
7\end{array}$ & $\begin{array}{l}100.71 \\
0\end{array}$ & $\begin{array}{l}100.69 \\
5 \\
\end{array}$ & $\begin{array}{l}100.68 \\
0\end{array}$ & $\begin{array}{l}100.66 \\
5\end{array}$ & $\begin{array}{l}100.65 \\
0\end{array}$ & $\begin{array}{l}100.63 \\
5\end{array}$ & $\begin{array}{l}100.62 \\
0\end{array}$ & $\begin{array}{l}100.61 \\
1\end{array}$ \\
\hline & 1860 & $\begin{array}{l}100.60 \\
4\end{array}$ & $\begin{array}{l}100.59 \\
0\end{array}$ & $\begin{array}{l}100.69 \\
5 \\
\end{array}$ & $\begin{array}{l}100.68 \\
0\end{array}$ & $\begin{array}{l}100.66 \\
5\end{array}$ & $\begin{array}{l}100.65 \\
0\end{array}$ & $\begin{array}{l}100.63 \\
5 \\
\end{array}$ & $\begin{array}{l}100.62 \\
0\end{array}$ & $\begin{array}{l}100.60 \\
5\end{array}$ & $\begin{array}{l}100.59 \\
0\end{array}$ & $\begin{array}{l}100.58 \\
1\end{array}$ \\
\hline I & $\begin{array}{l}1863.29 \\
7\end{array}$ & $\begin{array}{l}100.54 \\
2\end{array}$ & $\begin{array}{l}100.73 \\
0\end{array}$ & $\begin{array}{l}100.70 \\
5 \\
\end{array}$ & $\begin{array}{l}100.68 \\
0\end{array}$ & $\begin{array}{l}100.65 \\
5\end{array}$ & $\begin{array}{l}100.63 \\
0 \\
\end{array}$ & $\begin{array}{l}100.60 \\
5 \\
\end{array}$ & $\begin{array}{l}100.58 \\
0 \\
\end{array}$ & $\begin{array}{l}100.55 \\
5\end{array}$ & $\begin{array}{l}100.53 \\
0\end{array}$ & $\begin{array}{l}100.51 \\
6 \\
\end{array}$ \\
\hline
\end{tabular}

1. Final quantity of Dense Bituminous Macadam (DBM) layer which is to be used as profile correction layer is calculated using Simpsons Rule. Formula used are as follows:

\section{Area (at any given chainage or cross section)}

$$
=\sum\left[\frac{1}{2} X \text { distance between two consecutive chainage along width } X\right.
$$

$\{($ elevation difference between proposed and existing elevation at first chainage)

+ (elevation difference between proposed and existing elevation at second chainage)\}]

\section{Total Volume}

$=\sum\left[\frac{1}{2} X\right.$ distance between two consecutive chainage along length $X$

(Area computed at first chainage + Area computed at second chainage)] 


\section{CONCLUSIONS}

Not enough literature on the above subject is available in Indian conditions ${ }^{[4]}$. Guidelines regarding transition of transverse slope become very important especially when runway is under military use and speed of aircraft is very large. There is also an opportunity to develop simple software/tool which could make all corrections in profile of runways according to $\mathrm{ICAO}^{[1][2]}$ especially for situations like cross runway.

\section{REFERENCES}

1. International Civil Aviation Organization Annex 14 Volume I: "Aerodrome Design and Operations"

2. Bant Singh \& Srijit Biswas Fie, "Upgrading Standards of Riding Quality in Bituminous Concrete -A Case Study “, International Journal of Industrial Engineering\& Technology (IJIET), Vol. 3, Issue 3, pp. 21-34

3. International Civil Aviation Organization Aerodrome Design Manual (Doc 9157) Part 3: Pavements

4. Technical Instructions 2 of 1987 of Engineer - in - Chief's Branch New Delhi, Military Engineer Services

5. Ministry of Road Transport \& Highways: Specifications for Road and Bridge Works

6. N. Anusha \& P. Sai Avinash, "Experimental Investigation on Flexible Pavements with Various Soils ", International Journal of Civil Engineering ( IJCE), Vol. 6, Issue 6,pp. 61-72

7. Advisory Circular No 150/5300-13 date 09/29/89, "Airport Design" issued by Federal Aviation Administration (FAA) of US Department of Transportation. 